\title{
Análisis técnico-económico del sistema integrado por la producción de peces y la alimentación de aves con dietas elaboradas a partir de ensilado piscícolas
}

\author{
Jairo A. Camaño ${ }^{1}$, Luis F. Londoño² , y José E. Zapata ${ }^{1 \star}$ \\ (1) Facultad de Ciencias Farmacéuticas y Alimentarias. Grupo de Nutrición y Tecnología de Alimentos. Universidad de \\ Antioquia, Medellín, Colombia. (correo-e: jairo.camano@udea.edu.co; edgar.zapata@udea.edu.co) \\ (2) Facultad de Ciencias Agrarias, Politécnico Colombiano Jaime Isaza Cadavid, Grupo Gestiagro, Medellín, Colombia. \\ (correo-e: Iflondono@elpoli.edu.co)
}

* Autor a quien debe ser dirigida la correspondencia.

Recibido Mar. 30, 2021; Aceptado May. 28, 2021; Versión final Jun. 22, 2021, Publicado Oct. 2021

\begin{abstract}
Resumen
El principal objetivo de esta investigación es evaluar la viabilidad técnico-económica del sistema integrado por la producción de peces y la alimentación de aves con dietas elaboradas a partir de ensilado piscícolas mediante el análisis costo-beneficio. Se examinan cuatro sistemas de granjas: 1) producción de tilapia, 2) producción de tilapia y ensilados de sus vísceras, 3) producción de tilapia y aves alimentadas con materias primas convencionales y 4) producción de tilapias y aves alimentadas con ensilaje de vísceras y otras materias primas. Se determinaron parámetros como costos de inversión, producción, puntos de equilibrio, e indicadores financieros como el valor presente neto, tasa interna de retorno, relación beneficio-costo y periodo de recuperación de inversión. Los dos sistemas con producción de ensilados mostraron viabilidad económica significativa en comparación con los sistemas control. Se concluye que el aprovechamiento de las vísceras para producir ensilados como fuente de proteína para la alimentación de aves tiene viabilidad económica, además de ser una alternativa ambientalmente amigable.
\end{abstract}

Palabras clave: vísceras; tilapia; ensilado químico; análisis económico; piscicultura; avicultura

\section{Techno-economic analysis of a fish production and poultry feeding integrated system with a fish silage diet}

\begin{abstract}
The main objective of this research study is to conduct a techno-economic feasibility assessment and a costbenefit analysis of an integrated system of fish production and poultry feeding that uses diets made from fish silage. Four farm systems are examined: 1) tilapia production, 2) tilapia production and fish viscera silage, 3) tilapia production and poultry fed with conventional raw materials, and 4) tilapia production and poultry fed with viscera silage and other raw materials. The parameters determined included investment costs, production, and break-even points. The financial indicators evaluated included net present value, internal rate of return, benefitcost ratio, and payback period. The two systems with silage production show significant economic viability in comparison with the control systems. It is concluded that using viscera to produce silage as a protein source for poultry feed is economically viable and environmentally friendly.
\end{abstract}

Keywords: tilapia; viscera; chemical silage; economic analysis; fish farming; aviculture 


\section{INTRODUCCIÓN}

Actualmente el tratamiento de residuos sólidos es uno de los grandes desafíos a nivel global (You et al., 2016). Específicamente la industria acuícola, es uno de los sectores con mayor impacto en el ambiente (Rabassó y Hernández, 2015), lo que representa un problema importante si se tiene en cuenta que el rendimiento del filete esta entre el 30-50\% del pescado, dependiendo la especie (Arias et al., 2017) y para el año 2018, esta industria procesó aproximadamente 171 millones de toneladas de peces en el mundo, siendo considerado uno de los sectores industriales de mayor importancia económica y constante crecimiento a nivel global (FAO, 2018).

Algunos de los métodos que se han propuesto para el tratamiento de residuos acuícolas, apuntan a grandes productores industriales, lo que conlleva a que una gran cantidad de estos residuos sean desechados y vertidos de forma inadecuada por las pequeñas y medianas cooperativas piscícolas (Mota et al., 2019). Por su parte, el ensilaje de pescado ha sido utilizado en las últimas décadas como una tecnología alternativa para el procesamiento de residuos piscícolas (Davies et al., 2020). Este proceso consiste en el uso de ácidos orgánicos o inorgánicos, así como de algunas bacterias ácidos lácticas, para disminuir el pH y activar las enzimas endógenas, con el fin de provocar la lisis de las proteínas para favorecer la formación de péptidos y aminoácidos de interés, además, se provoca la disminución de la flora microbiana (Olsen et al., 2017). La principal característica de este tipo de productos radica en el contenido de proteína y su alta digestibilidad, por lo que ha ganado interés como materia prima proteica para la elaboración de dietas en diferentes especies de animales (Davies et al., 2020).

A pesar de que se han publicado un gran número de estudios asociados a las propiedades tecnológicas del ensilado de pescado y su utilización de ensilado de pescado como fuente de proteína para alimentación animal (Banze et al., 2017; Davies et al., 2020; Camaño et al., 2021), aún se carecen de estudios específicos de evaluación este proceso financiera o económicamente, que muestren la viabilidad económica de la implementación de un proceso industrial de ensilado de pescado acoplado a un sistema de granja piscícola convencional. Este es un aspecto relevante, puesto que el perfil económico de un proyecto productivo permite a las organizaciones o pequeños productores, la toma de decisiones financieramente correctas (Živković et al., 2017). Es así como diferentes investigaciones, han empleado enfoques piloto que permiten obtener la mayor información posible a un menor costo, sobre el perfil económico de un modelo productivo, con el cual se puedan hacer estimaciones a gran escala para respaldar decisiones de inversión (Farid et al., 2020).

Una de las herramientas financieras que han sido utilizadas para la evaluación económica de proyectos relacionados con el tratamiento de residuos, es el análisis de costo-beneficio (ACB) (Di Trapani et al., 2014). Mediante esta herramienta se busca estimar diferentes parámetros económicos tales como; el valor presente neto (VPN), la tasa interna de retorno (TIR), la relación beneficio-costo (RBC) y el periodo de recuperación (PR), los cuales han sido usados en la evaluación económica del uso de residuos agroindustriales (You et al., 2016; Khootama et al., 2018; Weber et al., 2020). Por lo tanto, el objetivo del presente trabajo fue aplicar el análisis costo- beneficio, para evaluar la viabilidad técnico-económica del sistema integrado por la producción de tilapia roja (Oreochromis spp.), la elaboración de ensilado químico a partir de sus vísceras y su uso en la alimentación de gallinas ponedoras de la raza Isa-Brown y de pollos de engorde de la raza Ross 508.

\section{METODOLOGÍA}

La presente investigación se realizó con base en pequeñas y medianas comunidades de piscicultores en Colombia, que cuentan con escasos recursos y limitaciones económicas, pero que, debido a su alto número son generadoras de grandes cantidades de vísceras, las cuales se disponen de forma incorrecta debido a los altos costos de tratamiento, por lo que se contaminan el suelo, los recursos hídricos y en general se impacta el ambiente (Villamil, et al., 2017). En este sentido, la metodología presenta varias subsecciones. Primero se detallan los sistemas de estudio y procesos productivos, seguido de las evaluaciones económicas, como estimación de costos, puntos de equilibrio, ingresos y utilidades netas, y finalmente se detallan el análisis costo-beneficio el cual incluye los indicadores financieros como VPN, TIR, RBC y PR.

\section{Sistemas de estudio}

Se simularon 4 sistemas productivos, de los cuales 2 se basaron en el aprovechamiento industrial de los residuos provenientes del eviscerado de una granja piscícola productora de tilapia roja (Oreochromis spp.), a partir de resultados de estudios piloto realizados previamente en el grupo que desarrolló el presente trabajo (Gaviria et al., 2020; Gaviria et al., 2021), siendo esta la información de partida sobre las variables productivas para evaluar la viabilidad técnico-económica. Mientras que los otros 2 sistemas, consistieron en sistemas controles convencionales. La tabla 1 muestra la descripción de los cuatros sistemas evaluados, mientras que los diagramas de los cuatro sistemas de granjas se muestran en la Fig. 1. Producción de tilapia (GPC), 
producción tilapia y ensilados de sus vísceras (GPEQ), producción de tilapia y aves alimentadas con materias primas convencionales (GPAC) y producción de tilapias y aves alimentadas con ensilaje y otras materias primas (GPAE). Los sistemas GPC y GPAC aplican la normatividad del manejo de residuos para industrias piscícolas (República de Colombia, 2005), mientras que los sistemas GPEQ y GPAE disponen de estas para la producción de ensilado químico (EQ).

Tabla 1: Descripción de los sistemas productivos de granjas evaluados.

\begin{tabular}{|l|l|}
\hline Sistema & Descripción y características \\
\hline Sistema GPC & $\begin{array}{l}\text { Granja piscícola control. Este sistema consistió en una granja piscícola convencional productora } \\
\text { de tilapia roja (Oreochromis spp.) sin aprovechamiento de residuos }\end{array}$ \\
\hline Sistema GPEQ & $\begin{array}{l}\text { Granja piscícola con producción de ensilado químico. Este sistema consistió en una granja } \\
\text { piscícola articulada a un sistema de producción y venta de ensilado químico seco y aceite de } \\
\text { pescado a partir de las vísceras producidas. }\end{array}$ \\
\hline Sistema GPAC & $\begin{array}{l}\text { Granja piscícola-avícola control. Este sistema se basó en la producción de tilapia y aves (gallinas } \\
\text { ponedoras de la raza Isa-Brown y de pollos de engorde de la raza Ross 508) alimentadas con } \\
\text { materias primas convencionales. }\end{array}$ \\
\hline Sistema GPAE & $\begin{array}{l}\text { Granja piscícola-avícola con producción de ensilado químico. Este sistema constó de una granja } \\
\text { piscícola relacionada con la alimentación de gallinas ponedoras de la raza Isa-Brown y producción } \\
\text { de pollos de engorde de la raza Ross 508, mediante dietas elaboradas a partir de ensilado } \\
\text { químico. }\end{array}$ \\
\hline
\end{tabular}

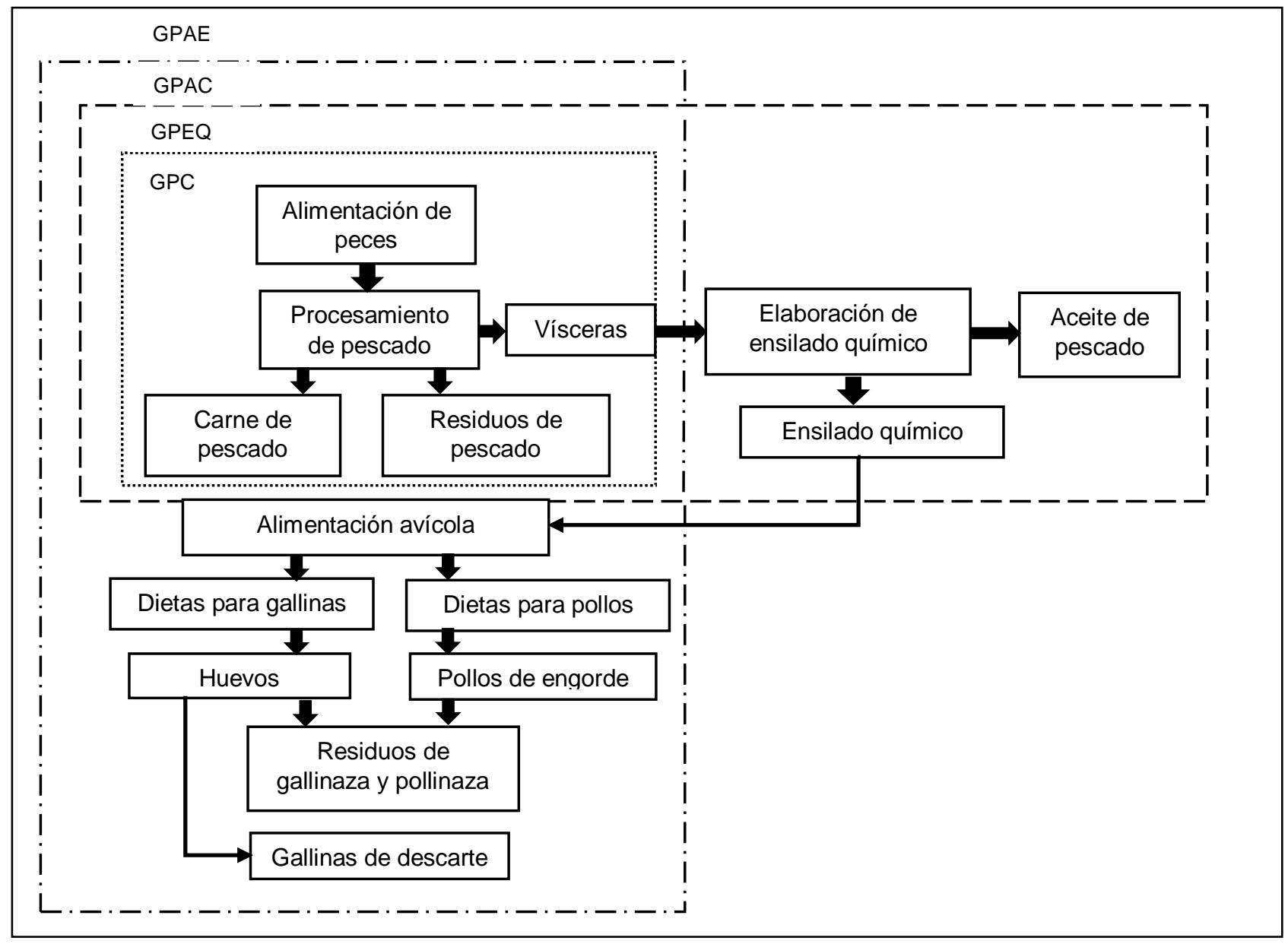

Fig. 1: Esquema de los diferentes sistemas productivos.

\section{Producción de peces}

Este sistema productivo fue definido con base en la literatura y consistió en el cultivo de 14308 alevinos de tilapia roja (Oreochromis spp.) durante 7 meses, de los cuales se espera obtener 12446 peces adultos, considerando una mortalidad del 13\% (Gonzales y Quevedo, 2018, FAO 2009). Los parámetros productivos de cosecha se obtuvieron mediante datos reportados por la FAO (FAO 2009), así como también los parámetros técnicos necesarios para las etapas de pre engorde y engorde. Este sistema requiere 3 tanques 
con geomembranas para la etapa de pre engorde durante 3 meses (densidad $=50$ peces $/ \mathrm{m}^{3}, \mathrm{~d}=4 \mathrm{~m}$ ) y 4 tanques para la etapa de engorde (densidad $=50$ peces $/ \mathrm{m}^{3}, \mathrm{~d}=6.5 \mathrm{~m}$ ) para los 4 meses restantes. La producción mensual de peces es de 1778 peces con masa promedio de $0.40 \mathrm{~kg}$ (FAO 2009). El contenido de vísceras producido mensualmente, se estimó teniendo en cuenta que el porcentaje de vísceras en tilapia varia de 12-18\% (Villamil et al., 2017), obteniéndose $113.8 \mathrm{~kg}$ (16\%) (Suarez et al., 2018), las cuales tendrán diferente disposición en función del sistema productivo de granja correspondiente.

\section{Producción de ensilado}

Las vísceras obtenidas son utilizadas para la elaboración de ensilado y aceite de pescado. Para esto se llevan a cabo las etapas de desengrasado, triturado, ensilaje y almacenamiento, según lo reportado por Suarez et al., (2018) y Camaño et al., (2020). El sistema GPEQ dispone de ensilado químico seco (EQS) y aceite de pescado para su venta, mientras que el sistema GPAE lo utiliza de forma humedad para su uso en la elaboración de dietas para la alimentación de aves. Se utilizó un $69 \%$ del ensilado producido para gallinas y un $29 \%$ para pollos, los cuales fueron definidos en función de las vísceras obtenidas y la cantidad requerida de alimentos, según la especie (Gaviria et al., 2020; Gaviria et al., 2021).

\section{Producción de dietas}

Consiste en la producción de alimento para gallinas ponedoras y pollos de engorde. Este proceso se aplica en los sistemas GPAE y GPAC, las cuales son granjas que contienen producción piscícola y avícola. Consta de tres etapas; mezclado, pelletizado y secado. Las formulaciones utilizadas, así como su respectiva composición nutricional fueron utilizadas de acuerdo con trabajos previos reportados por el grupo de investigación de los autores (Gaviria et al., 2020; Gaviria et al., 2021). Con el EQS obtenido en un mes (80 $\mathrm{kg}$ ) se elaboraron $116 \mathrm{~kg}$ de concentrado para gallinas, con una inclusión del $32 \%$ de ensilado en la dieta (Gaviria et al., 2020). Este alimento corresponde a la cantidad necesaria para 36 gallinas ponedoras durante un mes. Mientras que para la alimentación de pollos se elaboraron $90 \mathrm{~kg}$ de alimento, con una inclusión de 25.5\% de ensilado (Gaviria et al., 2021), obteniendo así lo requerido para 30 pollos de engorde durante un mes.

\section{Producción avícola}

Los parámetros utilizados para la producción de pollos y gallinas fueron establecidos según las experimentaciones llevadas a cabo por Gaviria et al., (2020); Gaviria et al., (2021), quienes evaluaron la producción de gallinas ponedoras y pollos de engorde respectivamente, mediante la inclusión de ensilado de vísceras de tilapia roja en las dietas, con las proporciones del presente estudio. Este sistema tiene 36 gallinas ponedoras de 18 semanas de edad y 30 pollos de engorde de 1 día de nacidos (Gaviria et al., 2020; Gaviria et al., 2021). Las aves son alimentadas dos veces al día, con suministro constante de agua, los pollos por 42 días, mientras que las gallinas por 1 año, periodo en el cual alcanzan el final de su vida útil y son vendidas. El ciclo productivo avícola corresponde a 7 meses, para los cuales se obtienen 150 pollos de peso promedio de 1.680 y $1.840 \mathrm{~kg}$ para el sistema GPAE y GPAC respectivamente (Gaviria et al., 2021), mientras que las gallinas producen 7182 y 6804 huevos por ciclo según las productividades reportadas por Gaviria et al., (2020) para los sistemas GPAE y GPAC respectivamente.

\section{Costos}

Los costos se diferenciaron en 3 categorías, costos de inversión (1), costos variables (2) y costos fijos (3). Para la estimación de los costos asociados a cada uno de los sistemas de granjas se tuvo en cuenta un ciclo productivo de 7 meses. 1) En este caso, para los 4 sistemas se calcularon los costos de inversión como la sumatoria de los costos de materiales, suministros y equipos que se deben tener para iniciar el ciclo productivo (Weber et al., 2020), tomando en cuenta información de proveedores y cotizaciones presupuestarias, incluyendo la mano de obra indirecta necesaria para la instalación de estructuras físicas, hidráulicas y redes eléctricas. Los costos (2) y (3) hacen referencia a los cotos de producción. 2) Los costos variables hacen referencia a todos los costos que están en función del volumen de producción, estos fueron calculados como la sumatoria de los costos de animales, materias primas, mano de obra directa, servicios industriales y costos indirectos de fabricación (CIF), los cuales incluyen materiales, suministros y otros (Arora et al., 2018). Los costos de transporte se asumieron despreciables con respecto a los otros costos variables involucrados, como ya han sido asumidos en estudios sobre el tratamiento de residuos (Bernstad y Jansen, 2011). 3) Los costos fijos fueron estimados como la sumatoria de los costos correspondientes a la depreciación de activos mediante el método de línea recta (Weber et al., 2020), para un periodo de 10 años (Mota et al., 2019), mantenimiento de equipos, el cual se consideró una tasa del 0.01 según You et al., (2016) y Weber et al., (2020), arrendamiento, gastos administrativos y de aseo. Todos los datos sobre precios y costos se basaron en el valor del mercado actual en pesos colombianos (COP) y fueron convertidos a dólares estadounidenses (USD), con una tasa de conversión adoptada de 3755.61 COP/ USD dada por el Banco de la República de Colombia. 


\section{Ingresos}

Los ingresos son el resultado de la venta de los productos obtenidos según el sistema de granja, se aplicó la metodología utilizada recientemente por Weber et al. (2020), determinando los precios promedio en el mercado para $1 \mathrm{~kg}$ de pescado, huevos (según su clasificación A, B ó C), $1 \mathrm{~kg}$ de pollo en pie, $1 \mathrm{~kg}$ de gallinaza y pollinaza compostada, $1 \mathrm{~kg}$ de aceite de pescado y gallinas de descarte de 70 semanas, mientras que para el ensilado químico seco se estimó según el costo del kg de proteína de la harina de pescado (\$0.82/g proteína) puesto que tienen digestibilidades similares y son fuente de proteína animal (Banze et al., 2017). Teniendo en cuenta que el costo de producción de $1 \mathrm{~kg}$ de EQS corresponde (0.58 USD) y que el precio de venta estimado fue de (0.80 USD), se obtendría una rentabilidad bruta del $37 \%$ aproximadamente.

\section{Estimación de puntos de equilibrio}

El punto de equilibrio (PE) proporciona un nivel de producción cuando los ingresos totales (IT) y los costos totales $(\mathrm{CT})$ son iguales (IT=CT) y no existe ganancia o pérdida. Este parámetro se estimó para cada producto siguiendo la metodología del margen de contribución ponderado (MCP), esta se fundamenta en el cálculo del margen de contribución para cada producto (MCPs) (Potkany y Krajcirova, 2015). En primer lugar, se estimó el porcentaje de participación en ventas para cada producto (\%PPs) con respecto al total de ventas (ecuación 1), seguido del cálculo del margen de contribución unitario (MCU) (ecuación 2). MCPs se calculó con la ecuación 3, mientras que MCP se estimó con la sumatoria de los MCPs. Finalmente, para calcular el punto de equilibrio en unidades totales $\left(\mathrm{PE}_{\mathrm{T}}\right.$ ) y el $\mathrm{PE}$ en unidades para cada producto ( $\mathrm{PE} \mathrm{E}_{\mathrm{S}}$ ) se emplearon las ecuaciones 4 y 5 respectivamente, donde CFT corresponde a los costos fijos totales.

$$
\begin{aligned}
& \% \mathrm{PP}_{\mathrm{S}}=\frac{\text { Ventas }_{\mathrm{S}}}{\text { Total }} \\
& \mathrm{MCU}=\mathrm{P}_{\mathrm{p} . \mathrm{u}}-\mathrm{CV}_{\mathrm{p} . \mathrm{u}} \\
& \mathrm{MCP}_{\mathrm{S}}=\mathrm{MCU} \% \mathrm{PP}_{\mathrm{S}} \\
& \mathrm{PE}_{\mathrm{T}}=\frac{\mathrm{CFT}}{\mathrm{MCP}} \\
& P E_{s}=P E_{S} . \% P P_{S}
\end{aligned}
$$

\section{Análisis costo-beneficio}

Este análisis se basa en el cálculo de indicadores financiero, estos indicadores son el resultado de la interacción de todos los componentes de un proyecto de inversión y son útiles para la evaluación, toma de decisiones (De Clercq et al., 2017) y para identificar los riesgos. Estos análisis se fundamentaron en un método factorial basado en la utilidad de ventas anual proyectas (Farid et al., 2020). El valor presente neto (VPV) se calculó según la ecuación 6 (You et al., 2016), donde la cantidad de periodos (LT) para lo cual se estimaron los flujos de caja una fueron 5 años, la entrada neta de efectivo $\left(\mathrm{C}_{t}\right)$ fue calculada actualizando los ingresos y egresos considerando una inflación promedio anual del 4.6\% (Banco de la República de Colombia, 2020), se emplearon los costos de inversión ( $C_{0}$ ) para cada sistema, la TMAR es la tasa mínima aceptable de retorno o también conocida como tasa de descuento, la cual fue estimada según la ecuación 7 . Donde i corresponde al premio al riesgo definido como el porcentaje de rendimiento que requieren los inversionistas y está en función del riesgo, para este caso se asignó un riesgo de medio correspondiente a un 10\%. Mientras que f corresponde a la inflación promedio anual. Esta tasa presentó valores similares ( $15 \%)$ a las tasas de descuentos reportadas por Manioğlu y Yilmaz (2006) para análisis económicos.

$$
V P N=\sum_{t}^{L T} \frac{C_{t}}{(1+T M A R)^{t}}-C_{o}
$$

Por otro lado, se estimó la tasa interna de retorno (TIR) la cual hace referencia al porcentaje de beneficio o pérdida que tendrá una inversión o proyecto, y corresponde a la tasa de descuento cuando se tiene un VPN de cero. Esta se calculó utilizando el algoritmo proporcionado por la herramienta informática Matlab (Licencia 914762, versión 2018). La relación beneficio-costo (RBC) se determinó según la ecuación 8 (You et al., 2016) incluyendo el costo de los egresos para el año $t\left(\mathrm{C}_{\mathrm{et}}\right)$. 
Para el análisis de estos parámetros se tuvieron en cuenta los criterios estándares (Weber et al., 2020). Si VPN $>0$, el modelo de granja es aceptado. Si VPN=0, la toma de decisiones es indiferente, mientras que si VPN $<0$ el modelo es rechazado. Mientras que si TIR $>$ TMAR, se acepta el modelo, si TIR $<$ TMAR se rechaza y si TIR=TMAR la toma de decisiones será indiferente. Para $R B C$ se tiene que sí $R B C>0$, se tiene un beneficio neto, y para $\mathrm{RBC}<0$ se tienen perdidas (You et al., 2016).

$$
\begin{aligned}
& \text { TMAR }=i+f+(i . f) \\
& R B C=\frac{V P N}{\sum_{t}^{L T} \frac{C_{e t}}{\left(1+\text { TMAR }^{t}\right.}+C_{o}}
\end{aligned}
$$

Finalmente, el periodo de recuperación de la inversión $(\mathrm{PR})$ hace referencia a la cantidad de años requeridos para que los flujos de efectivo descontados acumulados sean iguales a los costos totales e inversión inicial (Di Trapani et al., 2014). PR es una medida de qué tan pronto la inversión puede comenzar a generar ganancias, por lo que es deseable que sea lo más bajo posible (Engelberth, 2020). Este parámetro se calculó utilizando la ecuación 9, donde "A" corresponde al periodo anterior en el cual se recupera la inversión, "b" es el valor de la inversión inicial, "c" es el flujo de caja para el periodo "A" y "d" es el flujo de caja del periodo donde es recuperado la inversión.

$$
P R=A+\left[\frac{b-c}{d}\right]
$$

\section{RESULTADOS Y DISCUSIÓN}

Los resultados se presentan en varias secciones: En primer lugar, se detallan los resultados obtenidos para los costos de inversión y producción, seguido del análisis de ingresos, puntos de equilibrio y estado de resultado. Por último, se analizan los resultados del análisis costo-beneficio en donde se interpretan variables financieras como VPN, TIR, PR y RBC.

\section{Costos de inversión}

En la taba 2 y 3 se observan los costos asociados a los elementos iniciales de inversión para cada uno de los procesos involucrados en el análisis económico. A partir de estos se estimaron los costos totales de inversión para cada sistema evaluado en función de las etapas que involucra, como se presenta en la última columna de la tabla 4. El mayor costo en todos los sistemas, es el correspondiente a las instalaciones piscícolas, que además es la actividad principal. Esto se debe a los requerimientos de tanques en geomembranas que representan más del $50 \%$ de estos los costos. Por otro lado, se observa que el costo de inversión para GPC es menor en comparación a los otros 3 sistemas, debido a que esta granja es solo de producción piscícola, mientras que las otras involucran otros procesos adicionales. Por ejemplo, el sistema GPEQ incluye los cosos de inversión para la producción de ensilado, mientras que las granjas GPAE y GPAC abarcan tanto producción de ensilado, como producciones avícolas, incluyendo las dietas, presentando así los valores más altos.

\section{Costos de producción}

Los costos de producción (CP) para cada sistema productivo de granja se muestran en la tabla 3, los cuales representan la suma de los costos fijos y los variables. Se encontró que el sistema GPC y GPEQ son los que representa el costo más bajo debido a la simplicidad de las granjas, mientras que los sistemas GPAE y GPAC resultan tener los más altos costos, debido a la incorporación de sistemas productivos avícolas, que traen consigo un mayor número de actividades operativas y mayor utilización de recursos y servicios. La diferencia más significativa entre los sistemas GPEQ y GPC se observa en los servicios, por ejemplo, la granja GPC requiere la prestación de un servicio externo para la disposición final de las vísceras, según lo dispuesto por la normatividad colombiana en el decreto 838 de 2005 (República de Colombia, 2005), por un valor de 0.84 USD/ kg de residuo generado, elevando el valor en este concepto comparado con el sistema GPEQ, el cual aprovecha estos subproductos para la obtención de ensilado químico y aceite de pescado para su comercialización, como materias primas para dietas alimentarias avícolas, cambiando así el costo en un ingreso.

Por su parte, las diferencias entre GPAE y GPAC, se observan tanto en los servicios industriales como en las materias primas utilizadas, esto se debe al hecho que el sistema GPAC no aprovecha las vísceras y requiere el servicio industrial externo al igual que GPC y sumado a eso, utiliza mayores porcentajes en materias primas proteicas convencionales como harina de pescado y torta de soya, las cuales elevan el costo de producción para del alimento. 
Tabla 2: Costos de inversión (USD) para la estructura de granja piscícola y planta de procesamiento

\begin{tabular}{|c|c|c|c|}
\hline \multicolumn{4}{|l|}{ Granja piscícola } \\
\hline Concepto & Cantidad & Costo/u & Costo total \\
\hline Tanque G1 & 3 & 745.55 & 2236.65 \\
\hline Tanque G2 & 4 & 1011.81 & 4.047 .27 \\
\hline Sistema hidráulico & 1 & 84.40 & 84.40 \\
\hline Motobombas & 2 & 51.12 & 102.24 \\
\hline Filtros & 2 & 908.72 & 1817.45 \\
\hline Medidores de $\mathrm{pH}$ & 1 & 9.58 & 9.58 \\
\hline Sensores de $\mathrm{O}_{2}$ & 1 & 23.69 & 23.69 \\
\hline Bascula & 1 & 42.60 & 42.60 \\
\hline Canastillas & 6 & 2.39 & 14.37 \\
\hline Zorra mecánica & 1 & 50.59 & 50.59 \\
\hline Mesones & 2 & 50.59 & 101.18 \\
\hline Congelador & 1 & 958.56 & 958.56 \\
\hline Material de pesca & 1 & 61.24 & 61.24 \\
\hline Hidrolavadora & 1 & 79.88 & 79.88 \\
\hline Mano de obra & 1 & 88.61 & 88.61 \\
\hline \multicolumn{3}{|l|}{ Total } & 9718.38 \\
\hline \multicolumn{4}{|c|}{ Proceso de ensilado químico } \\
\hline Marmita a gas & 1 & 523.53 & 532.53 \\
\hline Pipeta de gas & 1 & 13.31 & 13.31 \\
\hline $\begin{array}{l}\text { Procesador de } \\
\text { alimentos }\end{array}$ & 2 & 16.77 & 33.54 \\
\hline Contenedores & 4 & 2.92 & 11.71 \\
\hline \multicolumn{3}{|l|}{ Total } & 590.56 \\
\hline \multicolumn{4}{|c|}{ Planta de procesamiento de dietas } \\
\hline Mezcladora & 1 & 505.90 & 505.90 \\
\hline Pelletizadora & 1 & 798.80 & 798.80 \\
\hline Secador solar & 1 & 69.22 & 69.22 \\
\hline Molino & 1 & 23.96 & 23.96 \\
\hline \multicolumn{3}{|c|}{ Total } & 1397.88 \\
\hline
\end{tabular}

Tabla 3: Costos de inversión (USD) para la estructura de granjas avícolas

\begin{tabular}{|l|c|c|c|c|c|}
\hline \multirow{2}{*}{ Concepto } & \multirow{2}{*}{ Unidad } & \multicolumn{2}{c|}{ Gallinas } & \multicolumn{2}{c|}{ Pollos } \\
\cline { 3 - 6 } & & Costo/u & Costo total & Costo/u & Costo total \\
\hline Tejas de zinc & Unidad & $4.39(4)$ & 17.56 & $4.39(2)$ & 8.78 \\
\hline Amarres & Paquete & $4.23(2)$ & 8.46 & $4.23(2)$ & 8.46 \\
\hline Tacos de acero & Unidad & $1.06(12)$ & 12.78 & $1.06(8)$ & 8.52 \\
\hline Malla de angeo & Metros & $0.47(30)$ & 14.37 & $0.47(20)$ & 9.58 \\
\hline Cortinas & Metros & $0.66(12)$ & 7.98 & $0.66(8)$ & 5.32 \\
\hline Bombillas & Unidad & $1.06(4)$ & 4.26 & $1.06(4)$ & 4.26 \\
\hline Otros & -- & -- & 45.35 & -- & 43.1 \\
\hline Jaulas & Unidad & -- & 157.09 & -- & -- \\
\hline Bascula & Unidad & -- & 3.99 & -- & -- \\
\hline Bebederos & Unidad & -- & -- & $1.78(1)$ & 1.78 \\
\hline Comederos & Unidad & -- & -- & $2.23(1)$ & 2.23 \\
\hline Compostaje & -- & -- & 6.11 & -- & 6.11 \\
\hline Mano de obra & -- & -- & 55.38 & -- & 55.38 \\
\hline Total & & \multicolumn{2}{|c|}{333.33} & & 153.57 \\
\hline
\end{tabular}

Tabla 4: Costos de inversión (USD) totales para cada sistema integrado de granja.

\begin{tabular}{|l|c|c|c|c|c|}
\hline \multirow{2}{*}{ Sistemas } & \multicolumn{5}{|c|}{ Costos de inversión (USD) } \\
\cline { 2 - 6 } & Piscícola & Ensilado químico & Dietas & Avícola & Inversión total \\
\hline GPC & 9718.38 & 0 & 0 & 0 & 9718.38 \\
\hline GPEQ & 9718.38 & 590.56 & 0 & 0 & 10308.94 \\
\hline GPAE & 9718.38 & 590.56 & 1397.88 & $333.33+153.57$ & 12193.72 \\
\hline GPAC & 9718.38 & 0 & 1397.88 & $333.33+153.57$ & 11603.16 \\
\hline
\end{tabular}


Es importante mencionar el alto porcentaje que representa el costo del alimento, con respecto a los costos totales de producción (tabla 5), con valores de 54.3, 58.9, 58.3 y 55.1\% para GPC, GPEQ, GPAE y GPAC, respectivamente. Estos valores fueron similares a los encontrados en otros estudios en sistemas piscícolas (Bozoglu y Ceyhan, 2009; Hadelan et al., 2012; Di Trapani et al., 2014). Se observa que los costos del alimento en GPEQ son muy similares a los de GPC, con una diferencia de $0.63 \%$, debido a que las materias primas para producir ensilado químico no representan un aumento significativo. Sin embargo, para el sistema GPAE, este costo se elevó en 566.45 USD (16\%) con respecto al modelo convencional GPC, debido a la inclusión de los costos de materias primas para la elaboración de las dietas para las aves. De igual forma, comparando GPAE respecto a GPAC se observa una disminución de 150.57 USD en los costos del alimento, acompañado de una disminución de 544.4 USD (72\%) para los costos de servicios, indicando que un piscicultor que desee incorporar especies avícolas mediante el sistema GPAE logrará menores costos de producción y en consecuencia mayores utilidades. Por su parte, el valor de las dietas se disminuye en un $19.98 \%$ (84.91 USD) y un $20.87 \%$ (65.66 USD) para la alimentación de gallinas y pollos respectivamente, cuando se utiliza ensilado químico en el sistema integrado GPAE, con respecto al sistema integrado convencional GPAC. Esto se debe a que la proteína de pescado representa el mayor de los costos de las materias primas para las dietas, el cual se reduce mediante la inclusión de 32 y $25.5 \%$ de ensilado, para el sistema GPAE, según lo reportado por Gaviria et al., (2020) y Gaviria et al., (2021).

Tabla 5: Costos fijos y variables (USD) para los diferentes modelos productivos por ciclo

\begin{tabular}{|c|c|c|c|c|c|c|c|c|}
\hline \multicolumn{9}{|c|}{ Costos fijos (CF) } \\
\hline Concepto & GPC & $\%$ & GPEQ & $\%$ & GPAE & $\%$ & GPAC & $\%$ \\
\hline Depreciación & 1123.47 & $81.2 \%$ & 1161.99 & $81.3 \%$ & 1261.45 & $81.3 \%$ & 1226.97 & $81 \%$ \\
\hline Mantenimiento & 112.34 & $8.1 \%$ & 120.05 & $8.4 \%$ & 139.94 & $8.4 \%$ & 133.04 & $9 \%$ \\
\hline Arriendo & 79.88 & $5.8 \%$ & 79.88 & $5.6 \%$ & 79.88 & $5.6 \%$ & 79.88 & $5.1 \%$ \\
\hline Administrativos & 13.65 & $1 \%$ & 13.65 & $1 \%$ & 15.47 & $1 \%$ & 15.47 & $1 \%$ \\
\hline Aseo & 54.00 & $3.9 \%$ & 54.00 & $3.8 \%$ & 61.18 & $3.8 \%$ & 61.18 & $3.9 \%$ \\
\hline Total & 1383.35 & $100 \%$ & 1429.57 & $100 \%$ & 1557.93 & $100 \%$ & 1516.56 & $100 \%$ \\
\hline \multicolumn{9}{|c|}{ Costos variables (CV) } \\
\hline Concepto & GPC & $\%$ & GPEQ & $\%$ & GPAE & $\%$ & GPAC & $\%$ \\
\hline Alimento-peces & 3587.64 & \multirow[t]{5}{*}{$54.3 \%$} & 3587.64 & \multirow[t]{5}{*}{$58.9 \%$} & 3587.64 & \multirow[t]{5}{*}{$58.3 \%$} & 3587.64 & \multirow[t]{5}{*}{$55.1 \%$} \\
\hline Alimento-gallinas & 0 & & 0 & & 340.06 & & 424.97 & \\
\hline Alimento-pollos & 0 & & 0 & & 248.95 & & 314.61 & \\
\hline Insumos EQ & 0 & & 22.65 & & 0 & & 0 & \\
\hline $\begin{array}{l}\text { SubTotal } \\
\text { Alimento, insumos }\end{array}$ & 3587.64 & & 3610.30 & & 4176.65 & & 4327.22 & \\
\hline Animales & 495.26 & $7.5 \%$ & 495.26 & $8.1 \%$ & 608.70 & $8.5 \%$ & 608.70 & $7.7 \%$ \\
\hline Mano de obra & 1780.93 & $26.9 \%$ & 1818.25 & $29.7 \%$ & 2097.60 & $29.3 \%$ & 2097.60 & $26,7 \%$ \\
\hline Indirectos & 27.95 & $0.4 \%$ & 30.88 & $0.5 \%$ & 78.05 & $1.1 \%$ & 78.05 & $1 \%$ \\
\hline Servicios & 720.73 & $10.9 \%$ & 177.42 & $2.9 \%$ & 203.95 & $2.8 \%$ & 748.36 & $9.5 \%$ \\
\hline Total & 6612.55 & $100 \%$ & 6132.14 & $100 \%$ & 7164.97 & $100 \%$ & 7859.95 & $100 \%$ \\
\hline $\begin{array}{l}\text { Costos de } \\
\text { producción total }\end{array}$ & \multicolumn{2}{|c|}{7995.90} & \multicolumn{2}{|c|}{7561.70} & \multicolumn{2}{|c|}{8722.90} & \multicolumn{2}{|c|}{9376.50} \\
\hline
\end{tabular}

Además, teniendo en cuenta que los costos de construcción de las instalaciones y los costos de operación son los que representan la mayor parte de los costos en estos sistemas y que la tasa de descuento y los otros conceptos de costos son menos controlables, se deben plantear estrategias que permitan la reducción de los costos operacionales y de construcción para permitir una mejora en la economía de los sistema evaluados, con especial atención en los valores de RBC para el aprovechamiento de vísceras mediante la producción de ensilado químico para alimentación de animales.

\section{Análisis de ingresos}

En la tabla 6, se observa el estado de resultado para los 4 sistemas de granjas correspondientes a un ciclo productivo (7 meses). Se obtuvo que las granjas GPEQ y GPAE presentan mayores ingresos y utilidades netas con respecto a las granjas control GPC y GPAC, debido a que el procesamiento de vísceras para la producción de ensilado químico y aceite de pescado, así como la producción avícola, generan nuevos ingresos a estos sistemas productivos. Además, el mayor ingreso y utilidad neta la obtuvo el sistema GPAE, seguido de GPEQ, mientras que los sistemas GPC y GPAC alcanzaron los valores más bajos, pero muy similares. A pesar de que los ingresos por venta de pescado en los sistemas GPEQ, GPAE y GPAC, 
correspondieron al $98.00,88.30$ y $89.29 \%$ respectivamente, se debe destacar que los sistemas GPEQ y GPAE aumentan las utilidades netas en un $18.52 \%$ (461.31 USD) y $22 \%$ (548.18 USD), respectivamente, con respecto a los sistemas convencionales GPC y GPAC. Mientras que un piscicultor que desee invertir en un pequeño sistema avícola usando materias primas convencionales, tan solo logrará un aumento del $0.1 \%$ en sus utilidades netas.

Estos resultados indican un alto potencial económico para la implementación de estos sistemas en pequeños y medianos productores, coincidiendo con afirmaciones realizadas por Mota et al. (2019), los cuales diseñaron un sistema de extracción de aceite de vísceras de tilapia nilótica en Brasil, concluyendo que el acoplamiento de un sistema que involucre procesos tecnológicos para el aprovechamiento tanto de la parte proteica como lipídica de las vísceras de pescado, pueden generar ingresos adicionales, minimizando los impactos ambientales generados por estos macro componentes orgánicos. Sin embargo, estos resultados no son suficientes para la determinación de la viabilidad económica de los sistemas evaluados, por lo que es necesario el análisis costo-beneficio (ACB).

Tabla 6. Estados de resultados para los diferentes sistemas productivos

\begin{tabular}{|l|c|c|c|c|c|c|c|c|}
\hline \multicolumn{1}{|c|}{ Concepto } & \multicolumn{2}{c|}{ GPC } & \multicolumn{2}{c|}{ GPEQ } & \multicolumn{2}{c|}{ GPAE } & \multicolumn{2}{c|}{ GPAC } \\
\hline Ingresos & & 11.553 .93 & & 11778.91 & & 13064.05 & & 12938.44 \\
\hline Pescado & 11.553 .93 & & 11553.93 & & 11553.93 & & 11553.93 & \\
\hline Aceite & -- & & 140.58 & & 140.58 & & -- & \\
\hline EQS & -- & & 84.13 & & -- & & -- & \\
\hline Huevos & -- & & -- & & 528.62 & & 503.10 & \\
\hline Gallinas & -- & & -- & & 27.96 & & 27.96 & \\
\hline Gallinaza & -- & & -- & & 241.55 & & 241.55 & \\
\hline Pollos & -- & & -- & & 437.18 & & 477.68 & \\
\hline Pollinaza & -- & & -- & & 134.19 & & 134.19 & \\
\hline$(-)$ CV & & 6612.55 & & 6132.30 & & 7164.97 & & 7859.95 \\
\hline Alimentos/Insumos & 3857.64 & & 3610.30 & & 4176.65 & & 4327.22 & \\
\hline Animales & 495.26 & & 495.26 & & 608.70 & & 608.70 & \\
\hline Mano de obra & 1780.93 & & 1818.25 & & 2097.60 & & 2097.60 & \\
\hline Indirectos & 27.95 & & 30.88 & & 78.05 & & 78.05 & \\
\hline Servicios & 720.73 & & 177.42 & & 203.95 & & 748.36 & \\
\hline Utilidades & & 4941.38 & & 5646.66 & & 5899.08 & & 5078.48 \\
\hline (-) CF & & 1833.35 & & 1429.61 & & 1557.93 & & 1516.56 \\
\hline Depreciación & 1123.47 & & 1161.99 & & 1261,45 & & 1.226 .97 & \\
\hline Mantenimiento & 112.34 & & 120.05 & & 139.94 & & 133.04 & \\
\hline Arriendo & 79.88 & & 79.88 & & 79.88 & & 79.88 & \\
\hline Administrativo & 13.65 & & 13.65 & & 15.47 & & 15.47 & \\
\hline Otros & 54.01 & & 54.01 & & 61.18 & & 61.18 & \\
\hline Utilidad & & 3558.02 & & 4217.05 & & 4341.14 & & 3561.92 \\
\hline Impuestos (-) & 1067.40 & & 1265.08 & & 1302.34 & & 1068.57 & \\
\hline Utilidad neta & & 2490.62 & & 2951.93 & & 3038.80 & & 2493.34 \\
\hline
\end{tabular}

\section{Análisis de puntos de equilibrio}

En la tabla 7, se observan los puntos de equilibrio de cada producto para cada sistema productivo, los cuales corresponden a las unidades que se necesita comercializar para cubrir los costos totales y no tener pérdidas en un ciclo de 7 meses. Se obtuvo que el producto que mayor contribuye a este aspecto es la venta de pescado, obteniéndose en todos los casos por encima de 1000 unidades. Este comportamiento es debido a que la actividad productiva principal en todos los sistemas estudiados es la piscícola, por un lado, por el volumen de producción y por otro porque es el punto de partida para las otras actividades productivas. Por su parte, se destaca que el sistema GPEQ requiere comercializar cantidades similares de pescado con respecto el sistema control GPC, mientras que el sistema GPAE las puede disminuir en 102 unidades, debido a la inclusión de más productos en venta como los huevos, pollos en pie y material compostado, por lo que se podría alcanzar el punto de equilibrio en menos tiempo e incluso generando mayores utilidades netas por ciclo como se mostró en la discusión anterior. Estos resultados demuestran que la rentabilidad de los sistemas integrados depende del tamaño de planta utilizada. 
Tabla 7. Puntos de equilibrio para los diferentes sistemas productivos

\begin{tabular}{|c|c|c|c|c|c|c|c|}
\hline \multirow{2}{*}{ Sistema } & \multicolumn{7}{|c|}{ Productos } \\
\cline { 2 - 8 } & $\begin{array}{c}K g \\
\text { Pescado }\end{array}$ & $\begin{array}{c}K g \\
\text { Aceite }\end{array}$ & $\begin{array}{c}K g \\
\text { EQS }\end{array}$ & $\begin{array}{c}\# \\
\text { Huevos }\end{array}$ & $\begin{array}{c}K g \\
\text { Gallinaza }\end{array}$ & $\begin{array}{c}K g \\
\text { Pollos }\end{array}$ & $\begin{array}{c}\mathrm{Kg} \\
\text { Pollinaza }\end{array}$ \\
\hline GPC & 1101 & -- & -- & -- & -- & -- & -- \\
\hline GPEQ & 1102 & 67 & 30 & -- & -- & -- & -- \\
\hline GPAE & 1003 & 132 & -- & 3598 & 454 & 168 & 336 \\
\hline GPAC & 1615 & -- & -- & 35091 & 4679 & 204 & 407 \\
\hline
\end{tabular}

De igual forma, se encontró que el sistema GPAC mostró valores superiores en comparación con los demás sistemas con ensilado, en cuanto a las unidades de pescado, huevos y $\mathrm{kg}$ de pollo, estas dos últimas producciones difíciles de alcanzar en un periodo de 7 meses, puesto que la capacidad de granja solo podría producir 6804 huevos y tan solo $276 \mathrm{~kg}$ de pollo en pie. Esto se debe principalmente a que se obtuvieron valores del margen de contribución unitario (MCU) negativos para estos productos, es decir, los costos variables unitarios son mayores a los precios de venta unitario, obteniéndose pérdidas económicas en el modelo avícola de este sistema. Debido a esto, se puede inferir que un pequeño piscicultor no podría sostener un sistema piscícola y avícola de esta capacidad utilizando materias primas convencionales para la alimentación de aves, mientras que si implementa el sistema GPAE puede llegar a la viabilidad económica. Este comportamiento se debe principalmente a que los sistemas GPEQ y GPAE presentan menores costos de producción que los sistemas control GPC y GPAC.

\section{Análisis costo-beneficio}

El análisis técnico-económico mediante la evaluación costo-beneficio (ACB) es una herramienta importante para validar la sostenibilidad y tomar decisiones en los sistemas de granjas estudiados, puesto que permite compararlos en términos de rentabilidad y otras variables técnico-económicas de importancia como son el VPN, la TIR, la RBC y el PR (tabla 8). Es así como el VPN que representan el total de los recursos que quedan a favor del sistema de granja al final del periodo (5 años en el presente estudio), en todos los sistemas de este estudio mostró valores positivos, siendo las granjas GPEQ y GPAE las que alcanzaron mayores valores de este parámetro. Es así como GPEQ tiene un VPN 39.56\% mayor que GPC. Mientras que GPAE tiene un $16 \%$ más, respecto a la misma granja GPC y $72 \%$ respecto a GPAC, reflejando las bondades de este sistema productivo.

Tabla 8. Evaluaciones financieras para los diferentes sistemas productivos

\begin{tabular}{|c|c|c|c|c|}
\hline Sistema & $V P N$ (USD) & TIR & $R B C$ & $P R$ (Años) \\
\hline GPC & 5741.82 & $37.30 \%$ & 0.087 & 2.07 \\
\hline GPEQ & 8013.77 & $43.70 \%$ & 0.123 & 1.50 \\
\hline GPAE & 6668.40 & $35.76 \%$ & 0.089 & 2.09 \\
\hline GPAC & 3873.68 & $28.09 \%$ & 0.050 & 2.21 \\
\hline
\end{tabular}

Por otro lado, los valores de TIR superaron el valor de TMAR en (15.15\%) para todos los casos, indicando que todos los sistemas de granja valorados pueden ser económicamente viables. No obstante, en cuanto a las utilidades netas y costos de producción del sistema GPAC se pudo determinar que la viabilidad está dada en mayor parte por la producción piscícola, puesto que quedó demostrado que el sistema avícola no genera utilidades, por lo que el modelo no sería realmente viable a comparación con el sistema convencional GPC y los sistemas basados en aprovechamiento de vísceras. A su vez, en los sistemas GPEQ y GPAE se lograron mayores valores de TIR que los sistemas control GPC y GPAC, siendo el sistema GPEQ el que presentó el mayor retorno económico (43\%). Este comportamiento también se observó para la RBC, donde los sistemas evaluados con el aprovechamiento de las vísceras con la tecnología de ensilaje químico (GPEQ y GPAE) obtuvieron mayores beneficios netos con respecto a las granjas control (GPC y GPAC), resaltando igualmente al sistema GPEQ como el más sobresaliente financieramente, debido a que permite un aumento del $3.6 \%$ y $7.3 \%$ de los beneficios netos en comparación con las granjas convencionales GPC y GPAC respectivamente.

Cabe mencionar que el sistema GPAE presenta valores similares a GPC en cuanto a RBC, pero superiores al modelo GPAC. Esto permite inferir que este último sistema es el que presenta menos desempeño productivo, con tan solo un $5 \%$ de beneficio neto, demostrando que el sistema multifuncional piscícola y avícola con materias primas convencionales, es el de menor desempeño entre los evaluados en este estudio. Los resultados obtenidos para el tiempo de recuperación de la inversión (PR) tuvieron un comportamiento 
similar a los parámetros VPN, TIR y RBC, para ambas granjas con inclusión de procesos de ensilados, mostrando claramente un tiempo de recuperación menor en comparación con las granjas control, siendo el sistema GPEQ el que permite recuperar la inversión en el mínimo tiempo, inclusive se reduce en un $25 \%$ comparado con los sistemas GPC y GPAE.

Para el sistema con mejor desempeño económico (GPEQ), se obtuvieron valores de $43 \%$ y 1.5 años, para TIR y PR, respectivamente. Valores igualmente buenos, fueron obtenidos por Mota et al., (2019) con TIR y PR de $45 \%$ y 1.9 años respectivamente, en el aprovechamiento de vísceras de tilapia nilótica (Oreochromis niloticus) para la extracción de aceite de pescado y su uso en la producción de biodiesel. Así mismo, en el aprovechamiento de residuos de batata con TIR y PR de 51\% 1.06 años, respectivamente (Weber et al., 2020). Sin embargo, algunos estudios de aprovechamiento de residuos con resultados inferiores a los del presente estudio, han sido considerados rentables y factibles económicamente, como es el caso de la producción de enzima lipasa de Aspergillus niger utilizando residuos agroindustriales, con TIR del 35\% (Khootama et al., 2018) y una biorrefinería de residuos de procesamiento de mango, con TIR de 34\% (Arora et al., 2018). Todos estos ejemplos indican que el aprovechamiento de residuos además de ser un ejercicio benéfico para el ambiente, puede llegar a ser viable económicamente. Sin embargo, también ha habido casos de aprovechamiento de residuos en los que no se ha llegado a la viabilidad económica, como es el caso la producción de metano a partir de residuos biológicos, cuyo VPN fue negativo (De Clercq et al., 2017) y una biorrefinería a base de biomasa de algas para la producción de biocombustibles y energía, que también fue inviable (Abdul y Lim, 2019). Estos resultados adversos indican que la viabilidad económica en los estudios con aprovechamiento de residuos, no está garantizada y que en cada caso se requiere hacer la evaluación.

Los resultados de los sistemas GPEQ y GPAE, señalan que se pueden obtener beneficios económicos, ya sea por la producción de EQS para su venta directa o mediante la implementación de un sistema avícola alimentado con el uso de EQ como materia prima proteica. Estos resultados aportan novedad debido a que en la actualidad es limitada la información acerca de evaluaciones financieras en procesos productivos que involucren ensilado de pescado o alguna otra forma de dar valor agregado a vísceras. Lo cual es pertinente debido a que el ensilado ha sido objeto de muchas investigaciones (Davies et al., 2020), y se ha caracterizado por tener un importante potencial, con alta viabilidad técnica en la sustitución de fuentes proteicas convencionales que son más costosas (Madage et al., 2015; Davies et al., 2020). Por último, se ha establecido que existen dificultades para realizar comparaciones con sistemas piscícolas en otros países debido a las particularidades de la piscicultura de cada nación, la cual es muy variante (Mota et al., 2019). Sin embargo, a pesar de que el análisis económico en el presente estudio fue realizado en función de las referencias de costos y precios recopilados del mercado colombiano, se puede destacar que el aprovechamiento de las vísceras de pescado mediante la tecnología de ensilado para su implementación en granjas piscícolas convencionales podría ser de gran utilidad económica y ambiental para cualquier productor en otros países.

\section{CONCLUSIONES}

De acuerdo con los resultados de este estudio se puede concluir que el aprovechamiento de las vísceras de tilapia roja producidas en las industrias piscícolas mediante la tecnología de ensilaje químico, es una alternativa económicamente viable ya sea para comercializar el ensilado seco o para alimentar aves en la misma granja.

\section{AGRADECIMIENTOS}

Los autores del presente trabajo agradecen a la Universidad de Antioquia y a COLCIENCIAS (proyecto 1115745-58746) por el apoyo financiero brindado para su desarrollo.

\section{NOTACION}

\section{Símbolos}

$\mathrm{ACB}=$ Análisis costo-beneficio

$\mathrm{C}_{\text {et }}=$ Costos de los egresos para el año $t$

$\mathrm{CV}_{\mathrm{p} \cdot \mathrm{u}}=$ Costos variable unitario

$\mathrm{C}_{0}=$ Costos de inversión

$\mathrm{LT}=$ Cantidad de periodos

$\mathrm{P}_{\text {p.u. }}=$ Precio por unidad de producto

$\mathrm{PE}=$ Punto de equilibrio

$P E_{s}=$ Punto de equilibrio para cada producto

$\mathrm{PE}_{\mathrm{T}}=$ Punto de equilibrio total

$\mathrm{PR}=$ Periodo de recuperación de inversión 
$\mathrm{PV}=$ Participación en ventas

$\mathrm{RBC}=$ Relación beneficio-costo

$\mathrm{TIR}=$ Tasa interna de retorno

TMAR= Tasa mínima aceptable de retorno

VPN= Valor presente neto

\section{Abreviaciones}

$\mathrm{CF}=$ Costos fijos

$\mathrm{CP}=$ Costos de producción

$\mathrm{CT}=$ Costos totales

$\mathrm{CV}=$ Costos variables

$\mathrm{EQ}=$ Ensilado químico

EQS = Ensilado químico seco

$\mathrm{GPC}=$ Granja piscícola convencional

GPAC = Granja piscícola y avícola convencional

GPAE = Granja piscícola y avícola con ensilado químico

GPEQ= Granja piscícola con ensilado químico

$\mathrm{IT}=$ Ingresos totales

$\mathrm{MCP}=$ Margen de contribución ponderado

$\mathrm{MCP}_{\mathrm{s}}=$ Margen de contribución ponderado para cada producto

\section{REFERENCIAS}

Abdul, N. N., y Lim, J.S., Evaluation of processing route alternatives for accessing the integration of algae-based biorefinery with palm oil mill, https://doi.org/10.1016/j.jclepro.2018.12.104, J. Clean. Prod., 212(2019), 1282-1299 (2019)

Arora, A., Banerjee, J., y otros tres autores., Process design and techno-economic analysis of an integrated mango processing waste biorefinery, https://doi.org/10.1016/j.indcrop.2018.02.061, Ind. Crops. Prod, 116(February), 24-34 (2018)

Banze, J.F., Da Silva, M.F.O., Enke, D.B.S., y Fracalossi, D.M., Acid silage of tuna viscera: Production, composition, quality and digestibility, https://doi.org/10.20950/1678-2305.2017.24.34, Bol. Inst. Pesca, 43, 24-34 (2017)

Bernstad, A., y Jansen, J., A life cycle approach to the management of household food waste - A Swedish full-scale case study, https://doi.org/10.1016/j.wasman.2011.02.026, Waste Manage, 31(8), 1879-1896 (2011)

Camaño, J.A., Rivera, A.M., y Zapata, J.E., Efecto del espesor de película y de la ubicación de la muestra en un secador solar directo, sobre la cinética de secado de ensilado de vísceras de tilapia roja (Oreochromis sp), https://doi.org/10.4067/s0718-07642020000100053, Inf. Tecnol, 31(1), 53-66 (2020)

Camaño, J.A.., Rivera, A.M., y Zapata Montoya, J.E., Sorption isotherms and thermodynamic properties of the dry silage of red tilapia viscera (Oreochromis spp.) obtained in a direct solar dryer, https://doi.org/10.1016/j.heliyon.2021.e06798, Heliyon, 7(4), e06798 (2021)

Davies, S.J., Guroy, D., y otros 3 autores., Evaluation of co-fermented apple-pomace, molasses and formic acid generated sardine based fish silages as fishmeal substitutes in diets for juvenile european sea bass (Dicentrachus labrax) production, https://doi.org/10.1016/j.aquaculture.2020.735087, Aquaculture, 521(September 2019), 735087 (2020)

De Clercq, D., Wen, Z., y Fei, F., Economic performance evaluation of bio-waste treatment technology at the facility level, https://doi.org/10.1016/j.resconrec.2016.09.031, Resour. Conserv. Recycl, 116, 178-184 (2017)

Di Trapani, A.M., Sgroi, F., Testa, R., y Tudisca, S., Economic comparison between offshore and inshore aquaculture production systems of European sea bass in Italy, https://doi.org/10.1016/j.aquaculture.2014.09.001, Aquaculture, 434, 334-339 (2014)

Engelberth, A., Evaluating Economic Potential of Food Waste Valorization: Onward to a Diverse Feedstock Biorefinery, https://doi.org/10.1016/j.cogsc.2020.100385, Curr. Opin. Green Sustain. Chem, 26, 100385 (2020)

FAO., The State of world fisheries and aquaculture, Meeting the Sustainable Development Goals, Roma, Italia (2018)

FAO., Oreochromis niloticus in cultured aquatic species fact sheets. Text by Rakocy, J. E. edited and compiled by Valerio Crespi and Michael New. CD-ROM (multilingual), Roma, Italia (2009)

Farid, M. A.A., Roslan, A.M., y otros cuatro autores., Net energy and techno-economic assessment of biodiesel production from waste cooking oil using a semi-industrial plant: A Malaysia perspective, https://doi.org/10.1016/j.seta.2020.100700, Sustain. Energy Technol. Assess, 39(March), 100700 (2020)

Gaviria, Y.S., Londoño, F.L.F., y Zapata, J.E., Effects of chemical silage of red tilapia viscera (Oreochromis spp.) as a source of protein on the productive and hematological parameters in isa-brown laying hens (Gallus gallus domesticus), https://doi.org/10.1016/j.heliyon.2020.e05831, Heliyon, 6(12) (2020) 
Gaviria, Y.S., Figueroa, O.A., y Zapata, J.E., Efecto de la inclusión de ensilado químico de vísceras de tilapia roja (Oreochromis spp.) en dietas para pollos de engorde sobre los parámetros productivos y sanguíneos, http://dx.doi.org/10.4067/S0718-07642021000300079, Inf. Tecnol, 32(3), 79-88 (2021)

Gonzales, C., y Quevedo, E., Cultivo de las tilapias roja (Oreochromis spp.) y plateada (Oreochromis nilotus) (p. 17), Pereira, Colombia (2018)

Hadelan, L., Par, V., Njavro, M., y Lovrinov, M., Real option approach to economic analysis of European sea bass (Dicetrarchus labrax) farming in Croatia, Agric. Conspec. Sci, 77(3), 161-165 (2012)

Khootama, A., Putri, D.N., y Hermansyah, H., Techno-economic analysis of lipase enzyme production from Aspergillus Niger using agro-industrial waste by solid state fermentation, https://doi.org/10.1016/j.egypro.2018.10.054, Energy Procedia, 153, 143-148 (2018)

Madage, S.S. K., Medis, W.U.D., y Sultanbawa, Y., Fish silage as replacement of fishmeal in red tilapia feeds, https://doi.org/10.1080/10454438.2015.1005483, J. Appl. Aquac, 27(2), 95-106. (2015)

Manioğlu, G., y Yilmaz, Z., Economic evaluation of the building envelope and operation period of heating system in terms of thermal comfort, https://doi.org/10.1016/j.enbuild.2005.06.009, Energy Build, 38(3), 266-272 (2006)

Mota, F.A.S., Costa, J.T., y Barreto, G.A., The Nile tilapia viscera oil extraction for biodiesel production in Brazil: An economic analysis, https://doi.org/10.1016/j.rser.2019.03.035, Renew. Sust. Energ. Rev, 108(March), 1-10 (2019)

Olsen, R.L., Toppe, J., y Karunasagar, I., Fish silage hydrolysates not only a feed nutrient, but also a useful feed aadditive, https://doi.org/10.1016/j.tifs.2017.06.003, Trends. Food. Sci. Tech, 66, 93-97 (2017)

Potkany, M., y Krajcirova, L., Quantification of the volume of products to achieve the break-even point and desired profit in non-homogeneous production, https://doi.org/10.1016/s2212-5671(15)00811-4, Procedia Econ. Financ, 26(15), 194$201(2015)$

Rabassó, M., y Hernández, J. M., Bioeconomic analysis of the environmental impact of a marine fish farm, https://doi.org/10.1016/j.jenvman.2015.04.034, J. Environ. Manage, 158, 24-35 (2015)

República de Colombia., Decreto 838 de 2005, Por el cual se modifica el Decreto 1713 de 2002 sobre disposición final de residuos sólidos y se dictan otras disposiciones, Bogotá, Colombia (2005)

Suarez, L.M., Montes, J.R., y Zapata, J.E., Optimización del contenido de ácidos en ensilados de vísceras de Tilapia roja (Oreochromis spp.) con análisis del ciclo de Vida de los alimentos derivados, https://doi.org/10.4067/s071807642018000600083, Inf. Tecnol., 29(6), 83-94 (2018)

Villamil, O., Váquiro, H., y Solanilla, J.F., Fish viscera protein hydrolysates: Production, potential applications and functional and bioactive properties, https://doi.org/10.1016/j.foodchem.2016.12.057, Food Chem, 224, 160-171 (2017)

Weber, C.T., Trierweiler, L.F., y Trierweiler, J.O., Food waste biorefinery advocating circular economy: Bioethanol and distilled beverage from sweet potato, https://doi.org/10.1016/j.jclepro.2020.121788, J. Clean. Prod, 268 (2020)

You, S., Wang, W., y otros tres autores., Comparison of the co-gasification of sewage sludge and food wastes and costbenefit analysis of gasification- and incineration-based waste treatment schemes, https://doi.org/10.1016/j.biortech.2016.07.017, Bioresour. Technol, 218, 595-605 (2016)

Živković, S.B., Veljković, M.V., y otros siete autores., Technological, technical, economic, environmental, social, human health risk, toxicological and policy considerations of biodiesel production and use,

https://doi.org/10.1016/j.rser.2017.05.048, Renew. Sust. Energ. Rev, 79(February), 222-247 (2017) 
